\title{
THE DOUBLE RAMIFICATION CYCLE AND THE THETA DIVISOR
}

\author{
SAMUEL GRUSHEVSKY AND DMITRY ZAKHAROV
}

\author{
(Communicated by Lev Borisov)
}

\begin{abstract}
We compute the classes of universal theta divisors of degrees zero and $g-1$ over the Deligne-Mumford compactification $\overline{\mathcal{M}}_{g, n}$ of the moduli space of curves, with various integer weights on the points, in particular reproving a recent result of Müller.

We also obtain a formula for the class in $C H^{g}\left(\mathcal{M}_{g, n}^{c t}\right)$ (moduli of stable curves of compact type) of the double ramification cycle, given by the condition that a fixed linear combination of the marked points is a principal divisor, reproving a recent result of Hain.

Our approach for computing the theta divisor is more direct, via test curves and the geometry of the theta divisor, and works easily over all of $\overline{\mathcal{M}}_{g, n}$. We used our extended result in another paper to study the partial compactification of the double ramification cycle.
\end{abstract}

\section{INTRODUCTION}

Let $\mathcal{M}_{g, n}$ denote the moduli space of smooth genus $g$ curves with $n$ labeled distinct marked points, let $\mathcal{M}_{g, n}^{c t}$ denote its partial compactification by stable curves of compact type, and let $\overline{\mathcal{M}}_{g, n}$ denote the Deligne-Mumford compactification by stable curves. Let $\mathcal{J}_{g}^{d} \rightarrow \mathcal{M}_{g}^{c t}$ denote the universal family of Picard varieties (Jacobians) of degree $d$ (recall that the Jacobian of a stable curve of compact type is in fact an abelian variety), and by abuse of notation let $\mathcal{J}_{g}^{d} \rightarrow \mathcal{M}_{g, n}^{c t}$ also denote its pullback under the forgetful map $\pi: \mathcal{M}_{g, n}^{c t} \rightarrow \mathcal{M}_{g}^{c t}$. For any collection of integers $\underline{d}=\left(d_{1}, \ldots, d_{n}\right) \in \mathbb{Z}^{n}$ of total degree $\operatorname{deg} \underline{d}:=d_{1}+\ldots+d_{n}=d$, define the section $s_{\underline{d}}: \mathcal{M}_{g, n} \rightarrow \mathcal{J}_{g}^{d}$ by $s_{\underline{d}}\left(C, p_{1}, \ldots, p_{n}\right):=\sum d_{i} p_{i} \in \operatorname{Pic}^{d}(C)$.

The degree $g-1$ universal Picard variety has the universal theta divisor $\Theta \subset$ $\mathcal{J}_{g}^{g-1}$. This divisor is defined as the image of the Abel map $\mathcal{C}_{g}^{g-1} \rightarrow \mathcal{J}_{g}^{g-1}$ of the $(g-1)$-fold symmetric product $\mathcal{C}_{g}^{g-1}$ given by $\left(C, p_{1}, \ldots, p_{g-1}\right) \mapsto \mathcal{O}_{C}\left(p_{1}+\cdots+\right.$ $\left.p_{g-1}\right)$. The degree 0 universal Picard variety has the zero section $z_{g}: \mathcal{M}_{g}^{c t} \rightarrow \mathcal{J}_{g}^{0}$. We denote by $T$ the universal symmetric theta divisor $T \subset \mathcal{J}_{g}^{0}$ trivialized along the zero section, i.e. analytically the zero divisor of the function $\theta(\tau, z) / \theta(\tau, 0)$.

The main result of this paper is the computations of the pullbacks of $\Theta$ and $T$ to $\overline{\mathcal{M}}_{g, n}$, with the answer given in Theorem 6

Received by the editors July 2, 2012 and, in revised form, January 25, 2013.

2010 Mathematics Subject Classification. Primary 14H10; Secondary 14H51.

The research of the first author was supported in part by the National Science Foundation under grant DMS-10-53313. 
Our motivation for computing the pullbacks of the theta divisors is that they can be used to compute the classes of natural geometric loci on the moduli space of curves, which have been studied recently. One example is a result proved (in cohomology) by Hain in Hai1] (see Section 2 for notation):

Theorem 1. For $\operatorname{deg} \underline{d}=0$, the class in $C H^{g}\left(\mathcal{M}_{g, n}^{c t}, \mathbb{Q}\right)$ of the pullback of the zero section of the universal Jacobian variety $\mathcal{J}_{g}^{0}$ is equal to

$$
\left[s_{\underline{d}}^{*} z_{g}\right]=\frac{1}{g !}\left[\frac{1}{2} \sum_{i=1}^{n} d_{i}^{2} K_{i}-\frac{1}{2} \sum_{P \subseteq I}\left(d_{P}^{2}-\sum_{i \in P} d_{i}^{2}\right) \delta_{0}^{P}-\frac{1}{2} \sum_{h>0, P \subseteq I} d_{P}^{2} \delta_{h}^{P}\right]^{g},
$$

where $d_{P}:=\sum_{i \in P} d_{i}$.

The cycle $s_{d}^{*} z_{g}$ is known as the double ramification cycle, and the question of computing its class is due to Eliashberg. Geometrically, it can be interpreted as the locus of curves admitting a map to $\mathbb{P}^{1}$ with ramification multiplicities being all the positive $d_{i}$ over 0 , and all the negative $d_{i}$ over $\infty$.

The relationship of the double ramification cycle to the pullback of the theta divisor is as follows. Let $p: \mathcal{X}_{g} \rightarrow \mathcal{A}_{g}$ denote the universal family of principally polarized abelian varieties, let $z_{g}$ denote the zero section of this family, and let $T \subset \mathcal{X}_{g}$ denote the universal symmetric theta divisor trivialized along the zero section. Then

$$
\left[z_{g}\right]=\frac{[T]^{g}}{g !} \in C H^{g}\left(\mathcal{X}_{g}, \mathbb{Q}\right) .
$$

Note that the pushforwards $p_{*}\left([T]^{k}\right)$ were considered by Mumford and studied in detail by van der Geer vdG99, but (1.1), in the Chow ring, follows from the existence of a multiplicative decomposition for $R p_{*} \mathbb{Q}$, proved by Deninger and Murre DM91] (see eg. [BL04, Cor. 16.5.7], or [Voi12, Prop. 4.3.6, Cor. 4.3.9]). In cohomology (1.1) was proven independently by Hain [Hai11, Prop. 8.1].

A closely related result is the following very recent theorem of Müller Mül12, which is nearly equivalent to computing the pullback of the degree $g-1$ theta divisor (our notation is slightly different; see Section 2 for details):

Theorem 2. For any $\underline{d}$ with $\operatorname{deg} \underline{d}=g-1$, and such that at least one $d_{i}$ is negative, define the following locus in $\mathcal{M}_{g, n}$ :

$$
D_{\underline{d}}:=\left\{\left(C, p_{1}, \ldots, p_{n}\right) \in \mathcal{M}_{g, n} \mid h^{0}\left(C, d_{1} p_{1}+\cdots+d_{n} p_{n}\right) \geq 1\right\},
$$

and let $\bar{D}_{\underline{d}}$ denote its closure in $\overline{\mathcal{M}}_{g, n}$. Then in $\operatorname{Pic}_{\mathbb{Q}}\left(\overline{\mathcal{M}}_{g, n}\right)$ the class of $\bar{D}_{\underline{d}}$ is equal to

$$
\begin{gathered}
{\left[\bar{D}_{\underline{d}}\right]=-\lambda_{1}+0 \cdot \delta_{i r r}+\frac{1}{2} \sum_{i=1}^{n} d_{i}\left(d_{i}+1\right) K_{i}-\frac{1}{2} \sum_{P \subseteq I}\left(d_{P}^{2}-\sum_{i \in P} d_{i}^{2}\right) \delta_{0}^{P}} \\
-\frac{1}{2} \sum_{h>0, P \subseteq I}\left(d_{P}-h\right)\left(d_{P}-h+1\right) \delta_{h}^{P}-\sum_{P \subseteq I_{+}, h>d_{P}}\left(h-d_{P}\right) \delta_{h}^{P},
\end{gathered}
$$

where $I_{+}:=\left\{i \in I \mid d_{i} \geq 0\right\}$.

Theorem 1 was first proved by Hain in April 2003, using normal functions. His proof became more widely known (and we became aware of it) in February 2011 with the appearance of Hain's preprint Hai11. Over the locus of curves with rational tails $\mathcal{M}_{g, n}^{r t}$, this result was reproved by Cavalieri, Marcus, and Wise [CMW11. 
in July 2011, using Gromov-Witten theory. We obtained a proof of Theorem 1 in June 2011, and discussed it with Hain and others at PCMI in July 2011, in particular correcting Hain's original formula, see Hai11. Theorem 2 was proved by Müller Mül12 in March 2012. We have decided to make our results available due to the continued interest in the problem.

Our method is more elementary and could also be applied in various similar situations to compute classes related to the theta divisor. In particular in our paper GZ12 we consider degenerations of abelian varieties and prove an extension of Theorem 1, and primarily of formula (1.1), to the universal family of semiabelic varieties of torus rank one, using our computation of the class $\left[s_{d}^{*} T\right]$ on $\overline{\mathcal{M}}_{g, n}$.

We calculate the class of the theta divisor using test curves. In Section 2 we describe a basis of test curves and a basis for $\operatorname{Pic}_{\mathbb{Q}}\left(\overline{\mathcal{M}}_{g, n}\right)$, and compute their intersection numbers. This is a standard method, and the calculations are standard, but we briefly summarize them for convenience and for future reference - the result is given in Proposition 3. The more interesting part is computing the intersections of the test curves with the pullback of the theta divisors $\Theta$ and $T$ under the map $s_{\underline{d}}$, which is done by using the properties of the theta function and the Abel-Jacobi map. This is the content of Proposition 5. Finally, in Theorem 6 we derive the formulas for the pullbacks of the theta divisors.

We follow the standard notation and conventions for working on the moduli of curves, referring for example to [Mum83, HM98, [ACG11] for known results, discussion, and further references.

\section{Divisors, test CURVEs, AND InTERSECTION NUMBERS}

In this section we describe a basis of divisor classes on $\overline{\mathcal{M}}_{g, n}$ and a collection of test curves, and we compute their intersection numbers. Our computation technique is quite standard, but we include it for the sake of completeness and for possible reference value.

Let $\pi: \overline{\mathcal{M}}_{g, n} \rightarrow \overline{\mathcal{M}}_{g}$ be the forgetful map and let $\pi_{i}: \overline{\mathcal{M}}_{g, n} \rightarrow \overline{\mathcal{M}}_{g, 1}$ be the map that forgets all but the $i$ th marked point. Let $I=\{1,2, \ldots, n\}$ denote the indexing set. For a subset $P \subseteq I$, let $P^{c}$ denote its complement. We consider the following set of generators for $\operatorname{Pic}_{\mathbb{Q}}\left(\overline{\mathcal{M}}_{g, n}\right)$ :

- The classes $K_{i}:=c_{1}\left(\pi_{i}^{*}\left(\omega_{p}\right)\right)$, where $\omega_{p}$ is the relative dualizing sheaf of the universal curve $p: \overline{\mathcal{M}}_{g, 1} \rightarrow \overline{\mathcal{M}}_{g}$.

- The classes $\delta_{h}^{P}$ of the boundary divisors $\Delta_{h}^{P}$, where $P \subseteq I$ and $0 \leq h \leq$ $g$. The generic point of $\Delta_{h}^{P}$ is a reducible curve consisting of a smooth component of genus $h$ containing the marked points indexed by $P$ and a smooth component of genus $g-h$ with the remaining points, joined at a node. To satisfy the stability condition we assume that $|P| \geq 2$ if $h=0$ and $|P| \leq g-2$ if $h=g$. Note that $\delta_{h}^{P}=\delta_{g-h}^{P^{c}}$. In any sum involving $\delta_{h}^{P}$, we assume that each divisor class appears only once, so we either explicitly state which one we are adding or we sum with symmetric coefficients. This convention is used by Müller Mül12 but not by Hain Hai11. We use $\delta$ to denote the divisor classes on the moduli stack and $\Delta$ to denote the divisors on the coarse moduli space.

- The class $\delta_{i r r}$ of the divisor $\Delta_{i r r}$. The generic point of $\Delta_{i r r}$ is a smooth curve of genus $g-1$ with two points identified to form a node.

- The first Chern class $\lambda_{1}$ of the Hodge bundle. 
The above classes are known to be a basis of $\operatorname{Pic}_{\mathbb{Q}}\left(\overline{\mathcal{M}}_{g, n}\right)$ for $g \geq 3$. In what follows we assume that $g \geq 3$, but our results also hold for $g=1$ and $g=2$ by inspection.

A more common choice of a basis replaces the classes $K_{i}$ with the classes $\psi_{i}$ of the cotangent bundles at the marked points $p_{i}$. We use the classes $K_{i}$ in our final result, but we use both $K_{i}$ and $\psi_{i}$ in intermediate calculations. These two classes differ by a linear combination of boundary classes (see [AC87, p. 161]):

$$
\psi_{i}=K_{i}+\sum_{P \ni i,|P| \geq 2} \delta_{0}^{P} .
$$

Note that the classes $K_{i}$ are denoted $\psi_{i}$ in [Hai11, while in [Mül12 the $\psi_{i}$ have the same meaning as above.

We now define a collection of test curves on $\overline{\mathcal{M}}_{g, n}$.

- The curves $Z_{i}$. Let $1 \leq i \leq n$; fix a generic smooth curve $\left(C, p_{1}, \ldots\right.$, $\left.\widehat{p}_{i}, \ldots, p_{n}\right) \in \mathcal{M}_{g, n-1}$. We define the family $Z_{i} \subset \overline{\mathcal{M}}_{g, n}$ by letting the point $p_{i}$ range over $C$. The curve $Z_{i}$ is isomorphic to $C$.

- The curves $Z_{h}^{P}$. Let $P=\left\{i_{1}, \ldots, i_{m}\right\} \subseteq I$ be a subset, let $P^{c}=$ $\left\{j_{1}, \ldots, j_{n-m}\right\}$ be the complement, and let $0 \leq h<g$, where we assume that $m \geq 2$ if $h=0$ and $m<n$ if $h=g-1$. Fix a generic smooth curve $\left(C_{1}, p_{j_{1}}, \ldots, p_{j_{n-m}}\right)$ in $\mathcal{M}_{g-h, n-m}$, and define the family $Y_{h}^{P} \subset \overline{\mathcal{M}}_{g-h, n-m+1}$ by adding a point $q_{1}$ and letting it range over $C_{1}$. Now fix another generic smooth curve $\left(C_{2}, p_{i_{1}}, \ldots, p_{i_{m}}, q_{2}\right) \in \mathcal{M}_{h, m+1}$, and define the family $Z_{h}^{P} \subset \overline{\mathcal{M}}_{g, n}$ by attaching $q_{1}$ to $q_{2}$ to form a node. The curves $Y_{h}^{P}$ and $Z_{h}^{P}$ are both isomorphic to $C_{1}$.

- The curve $\mathcal{E}$. Fix a generic smooth curve $\left(C_{2}, p_{1}, \ldots, p_{n}, q_{2}\right) \in \mathcal{M}_{g-1, n+1}$, and let $\mathcal{E}$ be the family obtained by attaching a varying elliptic curve $\left(C_{1}, q_{1}\right) \in \overline{\mathcal{M}}_{1,1}$ to the curve $C_{2}$ at $q_{2}$. We consider $\mathcal{E}$ to be "stacky", i.e. since $\left(C_{1}, q_{1}\right)$ has an involution, we consider the generic point of $\mathcal{E}$ with coefficient $1 / 2$.

- The curve $Z_{i r r}$. Fix a generic curve $\left(C_{1}, p_{1}, \ldots, p_{n}, q_{1}\right) \in \mathcal{M}_{g-2, n+1}$, and consider, for a fixed generic elliptic curve $\left(E, q_{2}\right)$ in $\mathcal{M}_{1,1}$, the family $Z_{i r r}$ obtained by varying a point $q_{3}$ over $E$, and attaching $q_{1}, q_{2}, q_{3}$ to a rational curve. The curve $Z_{i r r}$ is isomorphic to $E$.

We now compute, in the standard way, the intersection numbers of these test curves with the chosen basis of divisors, which will then imply that these curves form a basis for $N_{1}\left(\overline{\mathcal{M}}_{g, n}\right)$.

Proposition 3. The test curves have the following intersection numbers with the divisors, where we write $(P, h)=(Q, l)$ if $P=Q$ and $h=l$ or if $P^{c}=Q$ and $g-h=l$.

$$
\begin{gathered}
Z_{i} \cdot K_{j}=\left\{\begin{array}{cc}
2 g-2, & i=j, \\
0, & \text { otherwise },
\end{array} \quad Z_{i} \cdot \delta_{h}^{P}=\left\{\begin{array}{cc}
1, & (P, h)=(\{i, j\}, 0), j \neq i \\
0, & \text { otherwise },
\end{array}\right.\right. \\
Z_{i} \cdot \delta_{i r r}=0, \quad Z_{i} \cdot \lambda_{1}=0, \quad Z_{h}^{P} \cdot K_{i}=\left\{\begin{array}{cc}
2 g-2, & h=0 \text { and } i \in P, \\
1, & h>0 \text { and } i \notin P, \\
0, & \text { otherwise },
\end{array}\right. \\
Z_{h}^{P} \cdot \delta_{l}^{Q}=\left\{\begin{array}{c}
2-2(g-h)-\left|P^{c}\right|, \\
1, \\
0,
\end{array} \quad(Q, l)=(P, h),\right. \\
(P \sqcup\{j\}, h), j \in P^{c}, \\
\text { otherwise },
\end{gathered}
$$




$$
\begin{gathered}
Z_{h}^{P} \cdot \delta_{i r r}=0, \quad Z_{h}^{P} \cdot \lambda_{1}=0, \quad \mathcal{E} \cdot K_{i}=0, \quad \mathcal{E} \cdot \delta_{h}^{P}=\left\{\begin{array}{cc}
-1 / 24, & (P, h)=(\emptyset, 1), \\
0, & \text { otherwise },
\end{array}\right. \\
\mathcal{E} \cdot \delta_{i r r}=1 / 2, \quad \mathcal{E} \cdot \lambda_{1}=1 / 24, \quad Z_{i r r} \cdot \delta_{h}^{P}=\left\{\begin{array}{cc}
1, & (P, h)=(\emptyset, 1), \\
0, & \text { otherwise },
\end{array}\right. \\
Z_{i r r} \cdot K_{i}=0, \quad Z_{i r r} \cdot \delta_{i r r}=-1, \quad Z_{i r r} \cdot \lambda_{1}=0 .
\end{gathered}
$$

Proof. We first compute the intersections with the test curves $Z_{i}$. If $i \neq j$, then $\pi_{j}\left(Z_{i}\right)=\left\{\left(C, p_{j}\right)\right\}$ is a single point in $\mathcal{M}_{g, 1}$, so $K_{j}$ restricts to a trivial line bundle on $Z_{i}$ and $Z_{i} \cdot K_{j}=0$. On the other hand, $\pi_{i}\left(Z_{i}\right)$ is the fiber $\left\{\left(C, p_{i}\right) \mid p_{i} \in C\right\}$ of $\mathcal{M}_{g, 1}$ over $C \in \mathcal{M}_{g}$, so the bundle $K_{i}$ restricts to the cotangent bundle of $C$, which has degree $2 g-2$. The curves parametrized by $Z_{i}$ are smooth except when $p_{i}=p_{j}$ for some $j \neq i$, in which case the two marked points lie on a rational tail. This gives the intersection numbers with the boundary divisors. Finally, $\pi\left(Z_{i}\right)$ is a point, so the Hodge bundle is trivial on $Z_{i}$ and $Z_{i} \cdot \lambda_{1}=0$.

The remaining test curves are all supported on the boundary. To compute the intersection numbers with these curves we use the technique of $\S 2$ of [Fab99]. The boundary divisor $\Delta_{h}^{P}$ is the image of the map $\eta: \overline{\mathcal{M}}_{g-h, P^{c} \sqcup\left\{r_{1}\right\}} \times \overline{\mathcal{M}}_{h, P \sqcup\left\{r_{2}\right\}} \rightarrow$ $\overline{\mathcal{M}}_{g, n}$ identifying the marked points $r_{1}$ and $r_{2}$. We compute the intersection numbers with the test curves by pulling back to this product. We denote by $p r_{1}$ and $p r_{2}$ the projection maps to the two components. The pullback $\eta^{*} \psi_{i}$ is equal to either $p r_{1}^{*} \psi_{i}$ if $i \in P^{c}$ or $p r_{2}^{*} \psi_{i}$ if $i \in P$. According to [Fab99], the pullback $\eta^{*} \delta_{h}^{P}$ is either $-p r_{1}^{*} \psi_{r_{1}}-p r_{2}^{*} \psi_{r_{2}}+p r_{2}^{*} \delta_{g-2 h}^{I \sqcup\left\{r_{2}\right\}}$ if $g-2 h \geq 0$ and $P=I$ or $-p r_{1}^{*} \psi_{r_{1}}-p r_{2}^{*} \psi_{r_{2}}$ otherwise.

To compute $Z_{h}^{P} \cdot K_{i}$ for $h>0$, we note that the family $Z_{h}^{P}$ does not parametrize any curves with rational tails, so by (2.1) we have $Z_{h}^{P} \cdot K_{i}=Z_{h}^{P} \cdot \psi_{i}$. The preimage $\eta^{-1}\left(Z_{h}^{P}\right)$ is equal to $Y_{h}^{P} \times p t$, therefore $Z_{h}^{P} \cdot \psi_{i}=0$ if $i \in P$. If $i \in P^{c}$, then passing to the first factor in the product we have

$$
Z_{h}^{P} \cdot \psi_{i}=Y_{h}^{P} \cdot \psi_{i}=Y_{h}^{P} \cdot\left(K_{i}+\sum_{i \in Q \subseteq P^{c} \sqcup\left\{r_{1}\right\}} \delta_{0}^{Q}\right)=1,
$$

since $Y_{h}^{P} \cdot K_{i}=0$ as above, and the only boundary divisor in the sum that intersects $Y_{h}^{P}$ is $\delta_{0}^{\left\{p_{i}, r_{1}\right\}}$.

For the intersection numbers $Z_{0}^{P} \cdot K_{i}$, we note that the projection $\pi_{i}$ collapses rational tails, so the image $\pi_{i}\left(Z_{0}^{P}\right)$ is the point $\left(C_{2}, p_{i}\right) \in \overline{\mathcal{M}}_{g, 1}$ if $i \in P^{c}$, or the curve $\left\{\left(C_{2}, q_{1}\right) \mid q_{1} \in C_{2}\right\}$ if $i \in P$. Therefore, $Z_{0}^{P} \cdot K_{i}$ is zero in the first case and $2 g-2$ in the second.

The intersections of the test curves $Z_{h}^{P}$ with the boundary divisors correspond to the possible degenerations of the parameterized curves. All of the non-empty intersections are transverse and equal to one, except that the curve $Z_{h}^{P}$ lies on the divisor $\Delta_{h}^{P}$. Therefore, we again restrict to the first factor and obtain

$$
\begin{aligned}
& Z_{h}^{P} \cdot \delta_{h}^{P}=-Y_{h}^{P} \cdot \psi_{r_{1}}=-Y_{h}^{P} \cdot\left(K_{r_{1}}+\sum_{Q \subseteq P^{c}, Q \neq \emptyset} \delta_{0}^{Q \sqcup\left\{r_{1}\right\}}\right) \\
& =-Y_{h}^{P} \cdot K_{r_{1}}-\sum_{k \in P^{c}} Y_{h}^{P} \cdot \delta_{0}^{\left\{p_{k}, r_{1}\right\}}=-(2(g-h)-2)-\left|P^{c}\right| .
\end{aligned}
$$

Finally, the Hodge bundle on $Z_{h}^{P}$ is trivial, so $Z_{h}^{P} \cdot \lambda_{1}=0$. 
The curve $\mathcal{E}$ lies in the divisor $\Delta_{1}^{\emptyset}$ and intersects $\Delta_{i r r}$. The corresponding intersection numbers, as well as $\mathcal{E} \cdot \lambda_{1}$, were computed by Wolpert (see 4.15 and proof of Lemma 5.4 in Wol83]). Note, however, that for us $\delta_{1}^{\emptyset}$ and $\mathcal{E}$ are stacky with generic automorphism group of degree two, so the intersection numbers differ by factors of $1 / 2$ (see also [Mül12, Lemma 4.2]). Finally, for any $i$ the curve $\pi_{i}(\mathcal{E})$ is a fixed curve with a fixed marked point and an attached varying elliptic tail, so $\mathcal{E} \cdot K_{i}=0$.

The curve $Z_{i r r}$ lies in the boundary divisors $\Delta_{2}^{\emptyset}$ and $\Delta_{i r r}$, intersects $\Delta_{1}^{\emptyset}$ at one point, and does not intersect the other boundary divisors. To compute $Z_{\text {irr }} \cdot \delta_{2}^{\emptyset}$, we pull back to $\overline{\mathcal{M}}_{g-2, n+1} \times \overline{\mathcal{M}}_{2,1}$ as above. The divisor class $\delta_{2}^{\emptyset}$ pulls back to $-p r_{1}^{*} \psi_{r_{1}}-p r_{2}^{*} \psi_{r_{2}}$, and on the pullback of $Z_{i r r}$ both $r_{1}$ and $r_{2}$ are fixed points on fixed components, so $Z \cdot \delta_{2}^{\emptyset}=0$.

To calculate the intersection number $Z_{i r r} \cdot \delta_{i r r}$, we consider $\Delta_{i r r}$ as the image of the map $\xi: \overline{\mathcal{M}}_{g-1, n+2} \rightarrow \overline{\mathcal{M}}_{g, n}$ which glues together the last two marked points $r_{1}$ and $r_{2}$, and pull back to the moduli space $\overline{\mathcal{M}}_{g-1, n+2}$. According to Fab99, the pullback of $\delta_{i r r}$ to itself is

$$
\xi^{*} \delta_{i r r}=-\psi_{r_{1}}-\psi_{r_{2}}+\delta_{i r r}+\sum_{h=0 ; 1 \in Q \subseteq I ;(h, Q) \neq(g-1, I)}^{g-1} \sum_{h}\left(\delta_{h}^{Q \cup\left\{r_{1}\right\}}+\delta_{h}^{Q \cup\left\{r_{2}\right\}}\right) .
$$

Geometrically, the preimage of a point under $\xi$ is the normalization of the corresponding marked curve at a non-separating node. Therefore, the preimage $Y_{i r r}=$ $\xi^{-1}\left(Z_{i r r}\right)$ is a family that parametrizes reducible curves having three components: the fixed genus $g-2$ curve $\left(C_{1}, p_{1}, \ldots, p_{n}, q_{1}\right)$ containing the first $n$ marked points, an elliptic curve $\left(E, q_{2}, r_{2}\right)$ with $q_{2}$ fixed and $r_{2}$ varying, and a rational tail connecting $q_{1}$ and $q_{2}$, and containing $r_{1}$. To compute $Z_{i r r} \cdot \Delta_{i r r}$, we need to compute the intersections of $Y_{i r r}$ with the divisor classes on $\overline{\mathcal{M}}_{g-1, n+2}$ occurring in the above formula for $\xi^{*} \delta_{i r r}$.

The curve $Y_{i r r}$ lies in the boundary divisors $\delta_{1}^{\left\{r_{2}\right\}}=\delta_{g-2}^{I \cup\left\{r_{1}\right\}}$ and $\delta_{1}^{\left\{r_{1}, r_{2}\right\}}=\delta_{g-2}^{I}$, intersects at one point the boundary divisor $\delta_{1}^{\emptyset}=\delta_{g-2}^{I \cup\left\{r_{1}, r_{2}\right\}}$ (when $r_{2}$ hits $q_{2}$ it moves off $E$ onto a second rational bridge), and does not intersect the other boundary divisors. Also, it is clear that $Y_{i r r} \cdot \psi_{r_{1}}=0$.

Looking at the formula for $\xi^{*} \delta_{i r r}$, we only need to compute $Y_{i r r} \cdot \psi_{r_{2}}$ and $Y_{i r r}$. $\delta_{1}^{\left\{r_{2}\right\}}$. The computation of $Y_{i r r} \cdot \delta_{1}^{\left\{r_{2}\right\}}$ is similar to the ones we did previously. We pull back a second time via the map $\eta: \overline{\mathcal{M}}_{g-2, n+2} \times \overline{\mathcal{M}}_{1,2} \rightarrow \overline{\mathcal{M}}_{g-1, n+2}$ whose image is $\Delta_{1}^{\left\{r_{2}\right\}}$. Let $t_{1}$ and $t_{2}$ denote the points of attachment, then $\delta_{1}^{\left\{r_{2}\right\}}$ pulls back to $-p r_{1}^{*} \psi_{t_{1}}-p r_{2}^{*} \psi_{t_{2}}$. The preimage $\eta^{-1}\left(Y_{i r r}\right)$ is $p t \times X_{i r r}$, where $p t$ is a fixed point and $X_{i r r}$ is a family parametrizing the fixed elliptic curve $\left(E, r_{2}, t_{2}\right)$, with the moving point $r_{2}$. Therefore

$$
Y_{i r r} \cdot \delta_{1}^{r_{2}}=-X_{i r r} \cdot \psi_{t_{2}}=-X_{i r r} \cdot\left(K_{t_{2}}+\delta_{0}^{\left\{r_{2}, t_{2}\right\}}\right)=-1 .
$$

Similarly, we see that $Y_{i r r} \cdot \psi_{r_{2}}=0$. Indeed,

$$
\psi_{r_{2}}=K_{r_{2}}+\sum_{Q \subset I \cup\left\{r_{1}\right\}, Q \neq \emptyset} \delta_{0}^{Q \cup\left\{r_{2}\right\}},
$$

none of these boundary divisors intersect $Y_{i r r}$, and $K_{r_{2}}$ integrates to zero on $Y_{i r r}$ because it is an elliptic curve. Putting all this together, we get that $Z_{i r r} \cdot \delta_{i r r}=-1$.

Finally, the Hodge bundle on $Z_{i r r}$ is trivial, so $Z_{i r r} \cdot \lambda_{1}=0$, and $Z_{i r r} \cdot K_{i}=0$ because the marked points are all fixed on a fixed component. 
A straightforward computation (noting that the matrix of intersections above is close to being diagonal) shows that the matrix of intersections of our test curves with the chosen basis of $\operatorname{Pic}_{\mathbb{Q}}\left(\overline{\mathcal{M}}_{g, n}\right)$ is non-degenerate, and we thus get

Corollary 4. The curve classes $Z_{j}, Z_{l}^{Q}, \mathcal{E}$, and $Z_{i r r}$ generate over $\mathbb{Q}$ the group $N_{1}\left(\overline{\mathcal{M}}_{g, n}\right)$ of numerical equivalence classes of curves on $\overline{\mathcal{M}}_{g, n}$.

\section{THE ClASS OF THE THETA DIVISOR}

In this section, we compute the intersection numbers of the test curves defined in the previous section with the pullbacks $\left[s_{\underline{d}}^{*} \Theta\right]$ and $\left[s_{\underline{d}}^{*} T\right]$ of the theta divisors, and then prove the main theorems.

Proposition 5. For $\operatorname{deg} \underline{d}=0$, we have

$$
Z_{i} \cdot\left[s_{\underline{d}}^{*} T\right]=d_{i}^{2} g, Z_{h}^{P} \cdot\left[s_{\underline{d}}^{*} T\right]=d_{P}^{2}(g-h) .
$$

For $\operatorname{deg} \underline{d}=g-1$, we have

$$
Z_{i} \cdot\left[s_{\underline{d}}^{*} \Theta\right]=d_{i}^{2} g, Z_{h}^{P} \cdot\left[s_{\underline{d}}^{*} \Theta\right]=\left(d_{P}-h\right)^{2}(g-h)
$$

(we compute the intersections with $\mathcal{E}$ and $Z_{\text {irr }}$ separately).

Proof. We compute these intersection numbers by noting that the restrictions of $s_{d}$ to the test curves can be understood as Abel-Jacobi embeddings. We first compute, for $\operatorname{deg} \underline{d}=0$, the intersections with $\left[s_{\underline{d}}^{*} T\right]$.

Since $\pi\left(Z_{i}\right)=C$ is a single point in $\overline{\mathcal{M}}_{g}$, the image $s_{d}\left(Z_{i}\right)$ lies inside $\operatorname{Pic}^{0}(C)$. The restriction of $s_{\underline{d}}$ to $Z_{i}$ is therefore the composition of an Abel-Jacobi embedding $Z_{i} \rightarrow \operatorname{Pic}^{0}(C)$ and a multiplication by $d_{i}$ map on $\operatorname{Pic}^{0}(C)$. The theta function restricted to the Abel-Jacobi image of the curve has degree $g$, while the pullback under the multiplication has degree $d_{i}^{2}$ on divisors. Therefore we get $Z_{i} \cdot\left[s_{d}^{*} T\right]=d_{i}^{2} g$.

Similarly, the Jacobian variety of any curve parameterized by $Z_{h}^{P}$ is $\operatorname{Pic}^{0}\left(C_{1}\right) \times$ $\operatorname{Pic}^{0}\left(C_{2}\right)$. The limit of the Abel-Jacobi embedding is a more delicate issue. Indeed, note that the Abel-Jacobi mapping is naturally an embedding $C \rightarrow \operatorname{Pic}^{1}(C)$. Thus to have a map $C \rightarrow \mathrm{Pic}^{0}(C)$, we need to choose a basepoint for the embedding. In a family of curves degenerating to some $C_{1} \cup C_{2}$ with one node, the limit of the chosen base point must lie on both $C_{1}$ and $C_{2}$, for the limit of the Abel-Jacobi embedding to be well-defined. Therefore it must be the point $q_{1} \in C_{1}$, which is identified with $q_{2} \in C_{2}$ to form the node. For the case of $\operatorname{deg} \underline{d}=0$, we thus have in the limit

$$
\begin{gathered}
s_{\underline{d}}\left(\left(C_{1}, p_{j_{1}}, \ldots, p_{j_{n-m}}, q_{1}\right),\left(C_{2}, p_{i_{1}}, \ldots, p_{i_{m}}, q_{2}\right)\right) \\
=\left(\sum_{j \in P^{c}} d_{j} p_{j}+d_{P} q_{1}, \sum_{i \in P} d_{i} p_{i}-d_{P} q_{2}\right) \in \operatorname{Pic}^{0}\left(C_{1}\right) \times \operatorname{Pic}^{0}\left(C_{2}\right) .
\end{gathered}
$$

We recall that the theta function on a decomposable abelian variety is the product of the theta functions on the two factors. The point $q_{1}$ varies along the curve $C_{1}$ while $q_{2}$ is fixed, hence the second term is a constant map. The first term is the composition of the Abel-Jacobi embedding of $C_{1}$ with a multiplication by $d_{P}$, and thus has degree $d_{P}^{2}(g-h)$. 
The computations for the pullback $\left[s_{\underline{d}}^{*} \Theta\right]$, for the case $\operatorname{deg} \underline{d}=g-1$, are similar. First, the intersections with $Z_{i}$ are the same as for $\left[s_{d}^{*} T\right]$, as the degree 0 and $g-1$ Picard varieties of a curve with rational tails are isomorphic.

The intersection numbers of $Z_{h}^{P}$ with $\left[s_{d}^{*} \Theta\right]$ are not the same, because the limit of the Abel-Jacobi map in degree $g-1$ is different. As a smooth curve $C$ degenerates to a nodal curve $C_{1} \cup C_{2}$, the Jacobian variety $\operatorname{Pic}^{g-1}(C)$ becomes $\operatorname{Pic}^{g-h-1}\left(C_{1}\right) \times$ $\operatorname{Pic}^{h-1}\left(C_{2}\right)$. Therefore,

$$
\begin{gathered}
s_{\underline{d}}\left(\left(C_{1}, p_{j_{1}}, \ldots, p_{j_{n-m}}, q_{1}\right),\left(C_{2}, p_{i_{1}}, \ldots, p_{i_{m}}, q_{2}\right)\right) \\
=\left(\sum_{j \in P^{c}} d_{j} p_{j}+\left(d_{P}-h\right) q_{1}, \sum_{i \in P} d_{i} p_{i}-\left(d_{P}-h+1\right) q_{2}\right) \\
\in \operatorname{Pic}^{g-h-1}\left(C_{1}\right) \times \operatorname{Pic}^{h-1}\left(C_{2}\right),
\end{gathered}
$$

and by the same reasoning we obtain $Z_{h}^{P} \cdot\left[s_{d}^{*} \Theta\right]=\left(d_{P}-h\right)^{2}(g-h)$.

We are now ready to prove our main result.

Theorem 6. For $\operatorname{deg} \underline{d}=0$, the class $\left[s_{d}^{*} T\right] \in \operatorname{Pic}{ }_{\mathbb{Q}}\left(\overline{\mathcal{M}}_{g, n}\right)$ of the pullback of the universal symmetric theta divisor trivialized along the zero section is equal to

$$
\left[s_{\underline{d}}^{*} T\right]=0 \cdot \lambda_{1}+0 \cdot \delta_{i r r}+\frac{1}{2} \sum_{i=1}^{n} d_{i}^{2} K_{i}-\frac{1}{2} \sum_{P \subseteq I}\left(d_{P}^{2}-\sum_{i \in P} d_{i}^{2}\right) \delta_{0}^{P}-\frac{1}{2} \sum_{h>0, P \subseteq I} d_{P}^{2} \delta_{h}^{P} .
$$

We denote $d_{P}:=\sum_{i \in P} d_{i}$, and the last sum includes every boundary divisor class exactly once.

For $\operatorname{deg} \underline{d}=g-1$, the class $\left[s_{d}^{*} \Theta\right] \in \operatorname{Pic}_{\mathbb{Q}}\left(\overline{\mathcal{M}}_{g, n}\right)$ of the pullback of the universal theta divisor is equal to

$$
\begin{aligned}
{\left[s_{\underline{d}}^{*} \Theta\right]=-\lambda_{1}+} & \frac{1}{8} \delta_{i r r}+\frac{1}{2} \sum_{i=1}^{n} d_{i}\left(d_{i}+1\right) K_{i}-\frac{1}{2} \sum_{P \subseteq I}\left(d_{P}^{2}-\sum_{i \in P} d_{i}^{2}\right) \delta_{0}^{P} \\
& -\frac{1}{2} \sum_{h>0, P \subseteq I}\left(d_{P}-h\right)\left(d_{P}-h+1\right) \delta_{h}^{P} .
\end{aligned}
$$

Moreover, the class of the divisor $\bar{D}_{\underline{d}}$ considered by Müller Mül12] is expressed in terms of $\left[s_{\underline{d}}^{*} \Theta\right]$ as

$$
\left[\bar{D}_{\underline{d}}\right]=\left[s_{\underline{d}}^{*} \Theta\right]-\sum_{P \subset I_{+}, d_{P}<h}\left(h-d_{P}\right) \delta_{h}^{P}-\delta_{i r r} / 8,
$$

where $I_{+}:=\left\{i \in I \mid d_{i}>0\right\}$, thus reproving the formula in Theorem 2 ,

Proof. According to Corollary 4, the curve classes $Z_{j}, Z_{h}^{P}, \mathcal{E}$, and $Z_{\text {irr }}$ generate $N_{1}\left(\overline{\mathcal{M}}_{g, n}\right)$. Moreover, the test curves $Z_{j}$ and $Z_{h}^{P}$ have zero intersection with the divisors $\lambda_{1}$ and $\delta_{i r r}$. Therefore to calculate the coefficients of the divisors $K_{i}$ and $\delta_{h}^{P}$ in the theta divisors it suffices to intersect both sides of (3.3) and (3.4) with the test curves $Z_{j}$ and $Z_{h}^{P}$, using Propositions 3 and 5 , and to verify that they are equal. This is a tedious but straightforward calculation. 
Computing the remaining coefficients is somewhat more complicated, since the test curves $\mathcal{E}$ and $Z_{i r r}$ parametrize curves of non-compact type, and we need to understand the degeneration of the Abel-Jacobi map to such curves.

We first compute the remaining coefficients in $\left[s_{d}^{*} T\right]$. To calculate the intersection number $\mathcal{E} \cdot\left[s_{\underline{d}}^{*} T\right]$, note that a generic point of $\mathcal{E}$ is a smooth elliptic curve $C_{1}$ attached to a fixed genus $g-1$ curve $C_{2}$ with all the marked points on it. According to (3.1), the limit of the Abel-Jacobi map at that point is $\left(0, \sum d_{i} p_{i}\right) \in \operatorname{Pic}^{0}\left(C_{1}\right) \times \operatorname{Pic}^{0}\left(C_{2}\right)$, where the second term is constant. The value of the theta function trivialized along the zero section at all such points is the constant number $\theta\left(\tau, \sum d_{i} p_{i}\right) / \theta(\tau, 0)$, where $\tau$ is the period matrix of $C_{2}$, and we can choose the marked points $p_{i}$ so that it is non-zero. This number does not change as the elliptic curve degenerates, therefore, $\mathcal{E} \cdot\left[s_{\underline{d}}^{*} T\right]=0$.

The calculation of $Z_{i r r} \cdot\left[s_{d}^{*} T\right]$ is similar. Consider a reducible curve consisting of a smooth genus $g-2$ component $\left(C_{1}, p_{1}, \ldots, p_{n}, q_{1}\right)$ attached to a smooth genus two component $\left(C_{2}, q_{2}\right)$. The value of the theta function trivialized along the zero section at such a curve depends only on the position of the marked points on the first component. Any point in $Z_{i r r}$ lies in a one-parameter family consisting of such curves, hence the theta function is constant on $Z_{i r r}$ and $Z_{i r r} \cdot\left[s_{\underline{d}}^{*} T\right]=0$. Intersecting both sides of (3.3) with $\mathcal{E}$ and $Z_{i r r}$ and using Proposition 3 shows that the coefficient of $\delta_{i r r}$ in $\left[s_{d}^{*} T\right]$ is also zero.

Before finding the remaining coefficients of $\left[s_{d}^{*} \Theta\right]$, we first prove formula (3.5). Over the smooth locus $\mathcal{M}_{g, n}$ the classes $D_{\underline{d}}$ and $\underline{s}_{\underline{d}}^{*} \Theta$ coincide by definition. However, the theta function vanishes identically on certain boundary components, so the divisor $s_{\underline{d}}^{*} \Theta$ is reducible and contains some boundary components with multiplicities that we now compute.

Consider a boundary divisor $\Delta_{h}^{P}=\overline{\mathcal{M}}_{g-h, n-m+1} \times \overline{\mathcal{M}}_{h, m+1}$. The restriction of the theta function to a generic point of this divisor is given by formula (3.2). It may happen that on one of the two components all marked points have nonnegative weights. In this case the image of the divisor on that component lies entirely inside the theta divisor of the corresponding Jacobian. Assume without loss of generality that this happens on the second component. The order of vanishing of the theta function on such a boundary divisor is equal to $h-d_{P}$ by the Riemann theta singularity theorem (indeed, as per formula (3.2) for the Abel-Jacobi map on reducible stable curves we see that we are computing the multiplicity of the Riemann theta function at the specified effective divisor) to relate $\left[\bar{D}_{\underline{d}}\right]$ and $\left[s_{\underline{d}}^{*} \Theta\right]$ we need to subtract the corresponding multiple of $\delta_{h}^{P}$.

Along $\Delta_{i r r}$, the generic vanishing order of the theta function is equal to $1 / 8$. This can be seen by looking at the Fourier-Jacobi expansion of the theta function (see e.g., the lowest order term in Don87, Lemma 3.1.1]). We note that we are dealing with the actual universal theta function and not with the polarization on semiabelic varieties. Subtracting all the generic vanishing we get

$$
\left[\bar{D}_{\underline{d}}\right]=\left[s_{\underline{d}}^{*} \Theta\right]-\sum_{P \subset I_{+}, d_{P}<h}\left(h-d_{P}\right) \delta_{h}^{P}-\delta_{i r r} / 8,
$$

proving formula (3.5).

It remains to compute the coefficients of $\lambda_{1}$ and $\delta_{i r r}$ in $\left[s_{\underline{d}}^{*} \Theta\right]$. First, we intersect with the curve $\mathcal{E}$. Once all the generic vanishing is taken out, the restrictions of $\bar{D}_{\underline{d}}$ and of $s_{\underline{d}}^{*} T$ to the curve $\mathcal{E}$ are the same, as indeed on a stable curve whose 
normalization consists of two components of genera 1 and $g-1$, the degree $g-1$ and 0 Abel maps have the same behavior on the elliptic curve (for which the genus minus one is equal to zero). Thus we deduce from $s_{d}^{*} T \cdot \mathcal{E}=0$ also that $\left[\bar{D}_{\underline{d}}\right] \cdot \mathcal{E}=0$ (compare also to Mül12, Lemma 4.2]). Denoting the coefficients of $\lambda_{1}$ and $\delta_{i r r}$ in $\left[s_{d}^{*} \Theta\right]$ by $a$ and $b$, respectively, and using (3.5) and the intersection numbers from Proposition 3, we see that $a+12 b=1 / 2$.

To finish the computation of the class $\left[s_{d}^{*} \Theta\right]$, we thus need to find one more relation. Intersecting with the test curve $Z_{i r r}$ is tricky, as it requires having an explicit description of the behavior of the map $s_{d}$ on irreducible stable curves. This can be accomplished by a careful study of the Fourier-Jacobi expansion, but we take another approach. Consider the restriction of the class $\left[s_{\underline{d}}^{*} \Theta\right]$ to the image of the gluing map $i: \mathcal{M}_{2,1}^{c t} \times \overline{\mathcal{M}}_{g-2, n+1} \rightarrow \overline{\mathcal{M}}_{g, n}$. We note that on $\mathcal{M}_{2}^{c t}$ (and thus also by pullback on $\mathcal{M}_{2,1}^{c t}$ ) the class $\lambda_{1}$ is a boundary, namely $\lambda_{1}=\delta_{1}^{\emptyset} / 5$ (see [HM98], p. 171). Therefore, computing the pullback $i^{*}\left[s_{\underline{d}}^{*} \Theta\right]$ gives us the one extra condition that we need. Using the pullback formulas described in [Fab99, we see that

$$
i^{*}\left[s_{\underline{d}}^{*} \Theta\right]=p r_{1}^{*}\left(a \lambda_{1}+\psi\right)+p r_{2}^{*}(\cdots),
$$

where the second term is not relevant for us.

To compute this class geometrically, let $\mathcal{P i c}^{1}=\mathcal{X}_{2}$ be the universal Picard variety over $\mathcal{M}_{2}^{c t}=\mathcal{A}_{2}$, and consider the map $s_{1}: \mathcal{M}_{2,1}^{c t} \rightarrow \mathcal{P i c}^{1}$ defined by $s_{1}(C, p)=p \in$ $\operatorname{Pic}^{1}(C)$. We need to compute $s_{1}^{*} \theta$, where $\theta \subset \mathcal{P i c}^{1}$ is the universal theta divisor. The image of $s_{1}$ is exactly $\theta$, so we compute its pullback using the adjunction formula. As a map of course moduli spaces $s_{1}$ is injective, but as a map of stacks $s_{1}$ may have a non-trivial ramification divisor due to the presence of automorphisms.

The generic points of $\mathcal{M}_{2,1}^{c t}$ and of $\mathcal{P i c}^{1}$ have trivial automorphism groups. The moduli space $\mathcal{M}_{2,1}^{c t}$ has two divisors whose generic points have non-trivial automorphisms, namely the locus of Weierstrass points and the boundary divisor $\delta_{1}^{\emptyset}$. On $\mathcal{P i c}^{1}$, the images of these two divisors under $s_{1}$ are the locus of 2 -torsion points and the subfamily of the universal family on $\mathcal{A}_{1,1}$, the moduli space of products of elliptic curves, that is trivial on one of the factors. The generic automorphism group both of the source and the target divisor is $\mathbb{Z} / 2 \mathbb{Z}$ in each of these two cases, so the map $s_{1}$ is unramified in codimension one, and therefore by the adjunction formula

$$
K_{\mathcal{M}_{2,1}^{c t}}=\left.\left(K_{\mathcal{P i c}^{1}}+\theta\right)\right|_{\theta} .
$$

It is well-known (see [ACG11, Thm. 7.15]) that $K_{\mathcal{M}_{2}^{c t}}=13 \lambda_{1}-2 \delta_{1}^{\emptyset}$. Denoting by $p: \mathcal{M}_{2,1}^{c t} \rightarrow \mathcal{M}_{2}^{c t}$ the projection map, we see that the canonical class of $\mathcal{M}_{2,1}^{c t}$ is

$$
K_{\mathcal{M}_{2,1}^{c t}}=p^{*}\left(K_{\mathcal{M}_{2}^{c t}}\right)+c_{1}\left(\omega_{\mathcal{M}_{2,1}^{c t} / \mathcal{M}_{2}^{c t}}\right)=13 \lambda_{1}-2 \delta_{1}^{\emptyset}+\psi
$$

Similarly, let $\pi: \mathcal{X}_{g} \rightarrow \mathcal{A}_{g}$ denote the universal family of principally polarized abelian varieties. It is well-known that $K_{\mathcal{A}_{g}}=(g+1) L$, where $L=\operatorname{det} \mathbb{E}$ is the determinant of the Hodge bundle $\mathbb{E}=\pi_{*}\left(\omega_{\mathcal{X}_{g} / \mathcal{A}_{g}}\right)$. Therefore,

$$
K_{\mathcal{P i c}^{1}}=K_{\mathcal{X}_{2}}=\pi^{*} K_{\mathcal{A}_{2}}+c_{1}\left(\omega_{\mathcal{X}_{g} / \mathcal{A}_{g}}\right)=\pi^{*}(3 L)+\pi^{*} L=4 \pi^{*} L
$$


By definition $L$ pulls back to $\lambda_{1}$, so we finally obtain

$$
\left.\theta\right|_{\theta}=K_{\mathcal{M}_{2,1}^{c t}}-\left.K_{\mathcal{P i c}^{1}}\right|_{\theta}=\psi+13 \lambda_{1}-2 \delta_{1}^{\emptyset}-4 \lambda_{1}=\psi+9 \lambda_{1}-2 \delta_{1}^{\emptyset}=-\lambda_{1}+\psi,
$$

using that $5 \lambda_{1}=\delta_{1}^{\emptyset}$ on $\mathcal{M}_{2,1}^{c t}$ in the last step. It follows that the coefficient $a$ of the class $\left[s_{\underline{d}}^{*} \Theta\right]$ is equal to -1 , completing the proof.

Proof of Theorem 1. The theorem directly follows from formula (3.3) for $\left[s_{\underline{d}}^{*} T\right]$, restricted to the moduli space $\mathcal{M}_{g, n}^{c t}$ of curves of compact type over which the universal family $\mathcal{X}_{g} \rightarrow \mathcal{A}_{g}$ extends, and from formula (1.1) for the zero section.

\section{ACKnowledgment}

The second author would like to thank Maksym Fedorchuk for a number of useful discussions.

\section{REFERENCES}

[AC87] Enrico Arbarello and Maurizio Cornalba, The Picard groups of the moduli spaces of curves, Topology 26 (1987), no. 2, 153-171, DOI 10.1016/0040-9383(87)90056-5. MR895568 (88e:14032)

[ACG11] Enrico Arbarello, Maurizio Cornalba, and Phillip Griffiths, Geometry of algebraic curves. Volume II, with a contribution by Joseph Daniel Harris, Grundlehren der Mathematischen Wissenschaften, 268, Springer, Heidelberg, 2011. MR2807457(2012e:14059)

[BL04] Christina Birkenhake and Herbert Lange, Complex abelian varieties, 2nd ed., Grundlehren der Mathematischen Wissenschaften [Fundamental Principles of Mathematical Sciences], vol. 302, Springer-Verlag, Berlin, 2004. MR2062673 (2005c:14001)

[CMW11] Renzo Cavalieri, Steffen Marcus, and Jonathan Wise, Polynomial families of tautological classes on $\mathcal{M}_{g, n}^{r t}$, J. Pure Appl. Algebra 216 (2012), no. 4, 950-981, DOI 10.1016/j.jpaa.2011.10.037. MR2864866 (2012j:14019)

[DM91] Christopher Deninger and Jacob Murre, Motivic decomposition of abelian schemes and the Fourier transform, J. Reine Angew. Math. 422 (1991), 201-219. MR.1133323 (92m:14055)

[Don87] Ron Donagi, Big Schottky, Invent. Math. 89 (1987), no. 3, 569-599, DOI 10.1007/BF01388986. MR.903385 (88j:14052)

[Fab99] Carel Faber, Algorithms for computing intersection numbers on moduli spaces of curves, with an application to the class of the locus of Jacobians, New trends in algebraic geometry (Warwick, 1996), London Math. Soc. Lecture Note Ser., vol. 264, Cambridge Univ. Press, Cambridge, 1999, pp. 93-109, DOI 10.1017/ CBO9780511721540.006. MR1714822(2000m:14032)

[vdG99] Gerard van der Geer, Cycles on the moduli space of abelian varieties, Moduli of curves and abelian varieties, Aspects Math., E33, Vieweg, Braunschweig, 1999, pp. 65-89. MR.1722539 (2001b:14009)

[GZ12] Samuel Grushevsky and Dmitry Zakharov, The zero section of the universal semiabelian variety, and the double ramification cycle, Duke Math. J. 163 (2014), no. 5, 953-982. MR 3189435

[Hai11] Richard Hain, Normal functions and the geometry of moduli spaces of curves, Handbook of moduli, Vol. I, Adv. Lect. Math. (ALM), Int. Press., Somerville, MA, 2013, pp. 527-578. MR3184171

[HM98] Joe Harris and Ian Morrison, Moduli of curves, Graduate Texts in Mathematics, vol. 187, Springer-Verlag, New York, 1998. MR.1631825 (99g:14031)

[Mül12] F. Müller, The pullback of a theta divisor to $\mathcal{M}_{g, n}$, Math. Nachr. 286 (2013), no. 11-12, 1255-1266. MR3092284

[Mum83] David Mumford, Towards an enumerative geometry of the moduli space of curves, Arithmetic and geometry, Vol. II, Progr. Math., vol. 36, Birkhäuser Boston, Boston, MA, 1983, pp. 271-328. MR717614 (85j:14046) 
[Voi12] Claire Voisin, Chow rings, decomposition of the diagonal and the topology of families, Annals. of Math. Stud., 187, Princeton Univ. Press., Princeton, NJ, 2014. MR 3186044

[Wol83] Scott Wolpert, On the homology of the moduli space of stable curves, Ann. of Math. (2) 118 (1983), no. 3, 491-523, DOI 10.2307/2006980. MR.727702 (86h:32036)

Department of Mathematics, Stony Brook University, Stony Brook, New York 11794 3651

E-mail address: sam@math.sunysb.edu

Department of Mathematics, Stony Brook University, Stony Brook, New York 117943651

E-mail address: dvzakharov@gmail.com 